\title{
Equipment Safety in Renewable Energies Exploitation
}

\author{
B. Nsom ${ }^{1}$ and K. Bouchlaghem ${ }^{1,2}$ \\ ${ }^{1}$ Université de Bretagne Occidentale. LBMS EA 4325. IUT de Brest. BP 93169 \\ Rue de Kergoat. 29231 BREST Cedex (France) \\ Phone:+0033 298017223 , Fax:+0033 298017265 , e-mail: blaise.nsom@univ-brest.fr \\ ${ }^{2}$ Ecole Polytechnique de Sousse. Boulevard Khalifa Karoui-Sahloul \\ 4054 SOUSSE (Tunisia) \\ Phone:+00216 734 43667, Fax:+00216 732 43685, e-mail: bouchlaghemkarim@yahoo.fr
}

\begin{abstract}
This paper considers the dam-break problem in a horizontal smooth $1 \mathrm{D}$ channel, for the purpose of equipment safety in renewable energies exploitation. The fluid is water and it can be described by a Newtonian model, provided that the inertial effects be neglected versus the viscous ones in the momentum balance. Assuming the shallow water approximation, a non dimensional equation is built from the continuity and the Navier-Stokes equations in the limit of zero-inertia and it is solved analytically in two limits: short time and long time. These solutions are then combined into a single, universal model. Limitations of the model are examined by comparison to a converged finite difference numerical solution of the flow equation.
\end{abstract}

\section{Key words}

Dam failure, Finite difference method, Flow regimes, Shallow water approximation, Similar solution.

\section{Introduction}

Nowadays, Hydraulics which represents $90 \%$ of the sources of renewable energies used for producing electricity in the world is in progress mostly in the developing countries. Hydraulics is the only renewable energy which allows to make a stock of energy. Owing to the huge quantities of water retained behind dams, genuine energy reservoirs are available and ready to produce electricity at request. The bigger the dam is, the greater the pressure at its bottom is. Due to the system malfunction or an act of war (e.g. Dnieproghes, Ukraine in 1941), the dam can collapse. The water released downstream can destroy fields, goods, infrastructure and kill people and animals.

Since Ritter's original work on dam-break flow [1], many studies have been performed focusing on experiments, theory and numerical methods [2]. Dam-break flow has become a classical hydraulic problem with such a large complexity that a higher degree of reproduction of real conditions raises new studies. Consider a dam obstructing a horizontal smooth channel, dry downstream and with a given quantity of fluid upstream (with height $h_{0}$ ), contained between a fix plate and a dam. At initial time, the dam collapses and the fluid is released downstream (positive wave), while a negative wave propagates upstream (negative wave). From dam-collapse date to time where negative wave reaches the fix plate, Ritter [1] gives the so-called inertial solution, stating that the wave front advances with a constant speed of $2 \sqrt{g h_{0}}$, while the negative wave moves back with constant speed $\sqrt{g h_{0}}$. The fluid is water and the flow is described by the Navier Stokes and continuity equations, together with the non slip condition. Assuming the shallow water approximation, this system of equations leads to the Saint-Venant equations [3], a one-dimensional hyperbolic system. The complete hydrodynamic equations describing this unsteady flow in open channel were solved by Faure and Nahas [4], using the method of characteristics. Hunt [5], comparing one-dimensional turbulent flow model down a slope with its viscous counterpart, concluded that the viscous flow model gives the best description for debris flows. Indeed, these flows develop within a long domain, i.e. a domain of space that is much longer than it is wide, so short time behavior described by the previous studies are inappropriate to give a complete description of these natural flows. Natural flows generally erode their bed and transport sediments. For image analysis purpose, experimentalists generally slow down the flow by using viscous complex mixtures of water with diverse additive. Nsom et al.[6] and Nsom [7] performed an experimental study with glucose-syrup fluids characterized with adjustable viscosity and density. Hunt [5] built similarity solutions for such "geological flows" down a sloping 1D channel. Also, Schwarz [8] achieved a numerical study of viscous thin liquid films down an inclined plane. Solving free surface lubrication equations, including the effects of 
both gravity and surface tension, he states a scaling law for the prediction of finger-width.

In this work, a 1-D model is presented, aiming to provide practical laws, useful to engineers. Assuming the shallowwater approximation, equations of motion governing viscous dam-break flow are built and put in nondimensional form and the initial and boundary conditions are stated. Then, an analytical solution is presented both for short time and long time behavior. Zoppou and Roberts [9] tested the performance of 20 explicit schemes used to solve the shallow water wave equations for simulating the dam-break problem. Comparing results from these schemes with analytical solutions to the dam-break problem with finite-water depth and dry bed downstream of the dam, they found that most of the numerical schemes produce reasonable results for subcritical flows. So an explicit procedure was used here, which does not take into account turbulence generated by dam-break wave, as the flow develops over a dry smooth bed [10]. This numerical solution is compared with the expermental and analytical results obtained in [7].

\section{Problem statement}

\section{A. Equation of motion}

Let $h_{0}$ denote the height of fluid at negative time in a smooth horizontal rectangular channel, $g$ the gravity, $\rho$ and $\mu$ the fluid density and viscosity, respectively. Using a cartesian system of coordinates with the origin at the dam site, $x$-axis lying on the channel-length and the $\mathrm{z}$-axis in the increasing vertical direction (fig. 1).

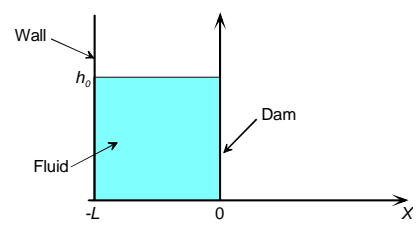

Fig 1: Configuration of horizontal dam-break flow at negative time

The fluid is assumed to flow mainly in the direction of $\mathrm{x}$ axis with height $h$ at the given control section of the abscissa $x$, at time $t$. So, the vertical velocities are negligibly small, and therefore the pressure is hydrostatic, the pressure in the flow is given by

$$
p=p_{0}+\rho g(h-z)
$$

where $p_{0}$ denotes the (constant) pressure at the free surface. The balance between the pressure gradient and the viscous forces is thus expressed by

$\frac{1}{\rho} \frac{\partial p}{\partial x}=g \frac{\partial h}{\partial x}=v \frac{\partial^{2} u}{\partial z^{2}}$

where the horizontal derivatives have been neglected in comparison with the vertical derivatives on the right-hand side of equ. (2) because the length of the current is very much greater than its thickness. At the base of the fluid layer the non slip condition writes $u(x, 0, t)=0$
Considering that the shear stress at the top of the current is very much less than its value within the current, it can be approximated as $\frac{\partial u}{\partial z}(x, h, t)=0$

the solution of equs. (2) - (4) is

$u(x, z, t)=-\frac{1}{2} \frac{g}{\nu} \frac{\partial h}{\partial x} z(2 h-z)$

A complete determination of the unknowns $u$ and $h$ requires the equation of continuity which can be written here as

$\frac{\partial h}{\partial t}+\frac{\partial}{\partial x}\left(\int_{0}^{h} u d z\right)=0$

Substituting (5) into (6) we obtain

$\frac{\partial h}{\partial t}-\frac{\rho g}{12 \mu} \frac{\partial^{2}\left(h^{4}\right)}{\partial x^{2}}=0$

If $l$ denotes the reservoir length, we can assume the following set of non dimensional variables:

$(H, X, T)=\left(\frac{h}{h_{0}}, \frac{x}{h_{0}}, \frac{\rho g h^{3}}{12 \mu l^{2}} t\right)$

where subscript $f$ denotes the wave-front, the equation of motion (7) then becomes, in the non dimensional form:

$\frac{\partial^{2}\left(H^{4}\right)}{\partial X^{2}}-\frac{\partial H}{\partial T}=0$

Equ. (9) is similar to the equation of motion obtained by Schwarz [10] and Barthes-Biesel [13], describing the evolution of a thin liquid layer flowing down a horizontal plane when surface tension effects can be neglected.

\section{B. Initial and boundary conditions}

Using (10), the fluid height at initial time is given by:

$$
H\left(X_{b}(T), T\right)=\left\{\begin{array}{cc}
1 & \text { for } \quad-1 \leq X \leq 0 \\
0 & \text { otherwise }
\end{array}\right.
$$

Furthermore, a complementary boundary condition should be imposed upstream, assuming that a short time or an asymptotic solution is sought. These boundary conditions are suggested by experimental observation. For the short time case, it is written as:

$$
H(X=-L, T)=1 \quad \text { with } \quad L=\frac{l}{h_{0}}
$$

which means that only a given fluid quantity in the upper part of the reservoir is released downstream the very few moments following the dam collapse.

While for the long time case, it is written as:

$$
\frac{\partial H}{\partial X}(X=-L, T)=0
$$

which means that there is no flow at the fixed wall; so at that site, the free surface is horizontal.

\section{Numerical solution}

\section{A. Equation of motion}


The problem to solve numerically is the same which has been solved analytically in the previous section by equs. (9)-(12). To build a numerical procedure, it is necessary to define the channel total length $l_{t}$. The non dimensional extreme (downwards) abscissa is $L_{e}=\frac{l_{l}-l}{h_{0}}$. This point is so far from dam site, that the flow is supposed to never reach it during a given experiment (1D assumption), with total duration $\tau$. This assumption constitutes the following complementary boundary condition: $\quad H\left(X_{e}, T\right)=0$ $\forall T \geq 0 \quad$ (13)

This problem is solved by a finite difference method. For this, the function $H(X, T)$ is computed in the set $\left[-L, L_{e}\right] \times[0, \tau]$, itself discretized in a finite number of identical small rectangles with sides $\Delta T$ and $\Delta X$. The equation will be approximated at grid points located at the following coordinates in the $[-L, L] \times[0, \tau]$ set:

$\left(X_{i}, T_{j}\right)=(-L+i \cdot \Delta X, j \cdot \Delta T)$

$i \in\left[0, \frac{-L+L_{e}}{\Delta X}\right\rfloor \quad, j \in\left[0, \frac{\tau}{\Delta T}\right\rfloor$

Notice that the equation of motion (9) can be put in the form:

$\frac{\partial H}{\partial T}=4 H^{3} \frac{\partial^{2} H}{\partial X^{2}}+3\left(\frac{\partial H^{2}}{\partial X}\right)^{2}$

An heuristic approach considers the product $\left(4 \mathrm{H}^{3}\right)$ in the right-hand side of eq.(38) as a "coefficient of diffusion" [16-18]. Indeed, the following equations are considered :

$\frac{\partial V}{\partial T}=4 \frac{\partial^{2} V}{\partial X^{2}} \quad, \quad V(X, 0)=H(X, 0)$

This numerical scheme is tested using the von Neumann method to provide a stability criterion which is necessary to ensure the convergence of our non-linear problem.

\section{B. Algorithms}

Using Taylor's formula, the derivative of the unknown function can be given by:

$$
\begin{aligned}
& \frac{\partial H}{\partial T}(X, T)=\frac{H(X, T+\Delta T)-H(X, T)}{\Delta T}-A, \\
& A=\sum_{n \geq 2} \frac{\Delta T^{n-1}}{n !} \frac{\partial^{n}}{\partial T^{n}}(X, T)
\end{aligned}
$$

Also, Taylor's formula can be used to write the non linear term in eq.(9):

$$
\begin{aligned}
& \frac{\partial^{2}\left(H^{4}\right)}{\partial X^{2}}(X, T)=B-\sum_{p \geq 2} \frac{\Delta X^{2(p-1)}}{(2 p) !} \frac{\partial^{2 p}\left(H^{4}\right)}{\partial X^{2 p}}(X, T) \\
& B=\underline{[H(X+\Delta X, T)]^{4}+[H(X-\Delta X, T)]^{4}-2[H(X, T)]^{4}}
\end{aligned}
$$

Introducing eq.(17) and eq.(18) in eq.(9) gives

$$
\begin{aligned}
& \frac{H(X, T+\Delta T)-H(X, T)}{\Delta T}=\frac{[H(X+\Delta X, T)]^{4}}{(\Delta X)^{2}}+C \\
& C=\frac{[H(X-\Delta X, T)]^{4}-2[H(X, T)]^{4}}{(\Delta X)^{2}}+R_{\Delta X, \Delta T}(X, T) \\
& \text { with } R_{\Delta X, \Delta T}(X, T)=\sum_{n \geq 2} \frac{\Delta T^{n}}{n !} \frac{\partial^{n} H}{\partial T^{n}}(X, T)-D
\end{aligned}
$$

$D=\Delta T \frac{\Delta X^{2(n-1)} \partial^{2 n}\left(H^{4}\right)}{(2 n) \cdot \partial X^{2 n}}(X, T)$

$R_{\Delta X, \Delta T}(X, T)$ is the residual term which is neglected to solve the numerical problem. Notice that this term can be numerically approximated knowing the solution at the former time step. Now let

$$
H_{i, j}=H\left(X_{i}, T_{j}\right)
$$

where $X_{i}$ and $T_{j}$ are given by eq.(34), then the finite difference equation to solve, which uses a first order time scheme and a centred second order spatial scheme, is written as

$$
H_{i, j+1}=H_{i, j}+\frac{\Delta T}{(\Delta X)^{2}}\left(\left[H_{i+1, j}\right]^{4}+\left[H_{i-1, j}\right]^{4}-2\left[H_{i, j}\right]^{4}\right)
$$

Notice that $H_{0, j+1}$ corresponds to upstream Neumann condition given by eq.(34). It is derived from eq.(21), say $H_{0, j+1}=H_{1, j+1}$. Also, if $i$ max denotes the maximum value that subscript $i$ can reach, i.e. $i \max$ is rounded off to the integer that is closest to $\frac{L+X_{e}}{\Delta X}$, then downstream Dirichlet condition given by eq.(35) yealds $H_{\text {imax }, j+1}=0$

In order to have a stability criterion, the equation (16) is discretized following the same numerical scheme, i.e. a first order time scheme and second order centred spatial scheme. The numerical problem is written :

$$
E=4 \frac{\Delta T}{(\Delta X)^{2}}\left(\left[V_{i+1, j}\right]+\left[V_{i-1, j}\right]-2\left[V_{i, j}\right)^{V_{i, j+1}=V_{i, j}+E}\right.
$$

with the same boundary conditions as $H$, i.e. : $V_{0, j+1}=V_{1, j+1}$ and $V_{i \max , j+1}=0$. Giving

$V_{p, q}=\sum_{k=0}^{i \max } \hat{V}_{k, q} e^{2 i \pi k p /(i \max +1)}$

with $\hat{V}_{k, q}$ the Fourier component corresponding to wave number $k$ at time $T=q \Delta T$, defined by:

$\hat{V}_{k, q}=\frac{1}{i \max +1} \sum_{p=0}^{i \max } V_{p, q} e^{-2 i \pi k p /(i \max +1)}$

the equation (45) is rewritten

$\hat{V}_{k, q+1}=\hat{V}_{k, q}\left(1-\frac{16 \Delta T}{\Delta X^{2}} \sin ^{2}(\pi k / i \max +1)\right)$

The stability criterion consists in considering that

$\left(1-\frac{16 \Delta T}{\Delta X^{2}} \sin ^{2}(k \pi / i \max +1)\right)<1, \forall k$

As $\sin ^{2}(k \pi / i \max +1)<1$, we obtain the following stability criterion:

$$
\frac{\Delta T}{\left(\Delta X^{2}\right)} \leq \frac{1}{8}
$$

We can notice that the numerical scheme described by equ. (22), makes $H_{I+1, j+1} \neq 0$ if $H_{I, j} \neq 0$. The front wave velocity, defined as $V_{f}=\frac{d X_{f}}{d t}$ must then verify

$V_{f} \leq \frac{\Delta X}{\Delta T}$

A McCormack finite difference scheme can improve the accuracy of the solution when $V_{e} \geq \frac{\Delta X}{\Delta T}$. In our case, the time step must be chosen small enough to verify the condition defined by equ. (28). 
horizontal.

\section{Results}

\section{A. Free surface profile}

The free surface profile is presented in fig.2. A large time after dam collapse, it completely differs from Ritter's solution, i.e. when the fluid is water, computed using equs.(1)-(2) which is concave. This shows that the convex shape of free surface profile for viscous dam-break flow is intrinsic to the equations of motion governing the problem. Furthermore, a complete description of the flow should include surface tension, introducing a complementary term in the equation of motion, say

$\frac{\partial H}{\partial T}=4 \frac{\partial}{\partial X}\left[H^{3} \frac{\partial H}{\partial X}-\frac{1 \partial^{3} H}{B} \partial X^{3}\right]$

where $\mathrm{B}$ denotes the Bond number, defined

as $B=\frac{\rho g L^{2}}{\sigma} \quad$ and $\sigma$ the fluid surface tension. Computation of equ. (30) was carried out using the procedure described in previous section for assigned glucose syrup concentration in water. Fluid physical properties (density, viscosity and surface tension) were taken in [19]. For similar flow configuration, results were quite identical to those obtained from equ. (9), i.e. when surface tension is neglected. In fact, surface tension would affect viscous dam-break flow, only in film lubrication conditions [10].

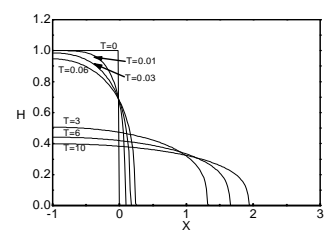

Figure 2: Time variation of free surface profile

\section{B. Fluid height}

Fig. 2 also shows that during a very short while after dam collapse (short time solution), the flow height remains constant at dam site, with

$H_{d}(X=0, T) \approx 0.684$

in excellent agreement with the analytical solution (equ.23).

The viscous solution is characterized by a decreasing of the fluid height at dam site. At a given location inside the reservoir, time variation of the fluid height is shown in fig. 3 which indicates that the fluid height collapses for stations close to dam site followed by a smoother decrease for all upstream stations. While at given downstream station, flow height increases abruptly at first stage, then smoothly to a maximum value and finally decreases as shown in fig. 4

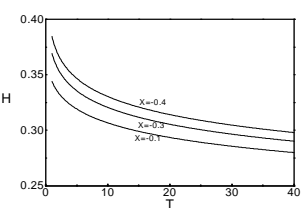

Figure 3: Typical time variation of fluid height at upstream stations

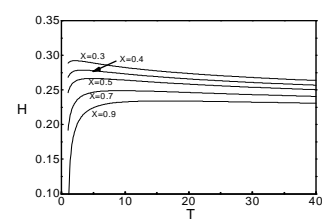

Figure 4: Typical time variation of fluid height at downstream stations

\section{Maximum heights}

To localize the maximum height at given down scenario described in Fig. 4 shows that

$$
\frac{\partial H(X, T)}{\partial T}=0
$$

While in the long time regime described by equ. (58), we have

$\frac{\partial H}{\partial T}=-\left[\frac{\left(X_{f}+1\right)^{\prime}}{\alpha_{m}\left(X_{f}+1\right)^{2}}\right]\left(\Psi+\lambda \Psi^{\prime}\right)$

So

$\Psi+\lambda \Psi^{\prime}=0$

which gives

$\lambda=\left(\frac{3}{5}\right)^{1 / 2}$

Introducing this solution in equ. (31), the maximum height at given downstream station $X$ is then found as $H_{\max }(X)=\left(\frac{2}{5}\right)^{1 / 3}\left(\frac{3}{5}\right)^{1 / 2} \frac{1}{\alpha} \frac{1}{\alpha+1)}$

The corresponding graph (hyperbola) is shown on Fig. 5

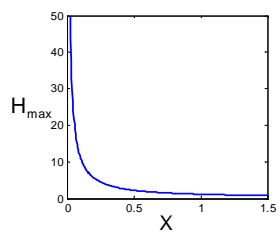

Figure 5: Variation of the maximum height at given station, vs the corresponding abscissa

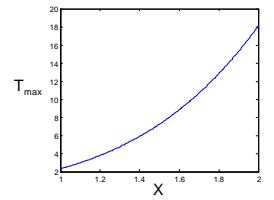

Figure 6: Variation of the time of occurrence of maximum height for given abscissa

and it occurs at time $T_{\max }$ such that

$T_{\max }(X)=\frac{1}{\gamma^{5}}\left(\frac{5}{3}\right)^{5 / 2}(X+1)^{5}+T_{c}-\left(\frac{1}{\gamma \alpha}\right)^{5}$

whose graph is shown on Fig. 6 


\section{Wave front position}

Time evolution of the front of the positive wave is presented in fig.7. It can be obtained either numerically (section 3) or analytically using equ. (20). This graph agrees with the experimental result obtained by Nsom [9] who found the following scaling law in this regime $X_{f} \propto T^{1 / 2}$

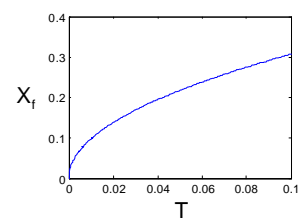

Figure 7: Evolution of the front of the positive wave front in short time viscous regime

In the same flow regime, the front of the negative wave, obtained numerically or analytically using equ. (19) is shown on fig. 8. It can be observed that $X_{b}(T)$ decreases vs time with a slope itself decreasing in the time, while for long time flow regime, the graph of the equation of motion of the front wave is shown on fig. 9. It can be obtained numerically (section 3) or analytically using equ. (28) and this result agrees with the experimental result obtained by Nsom [9] who found the following scaling law in this regime

$X_{f} \propto T^{1 / 5}$

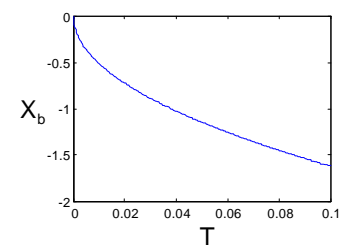

Figure 8: Evolution of the front of the positive wave front in long time viscous regime

In these experiments, the author performed dam-break tests using well characterized water-glucose syrup solutions. Generally, in the literature, theoretical studies focus on the long time solution also called asymptotic solution (e.g.: [7]) and the scaling law obtained is of the form of equ.(38). The originality of the present paper is to point out for the first time numerically, the previous two viscous flow regimes and to characterize them.

\section{E. Wave front velocity}

The wave front velocity is obtained from the time derivation of $X_{f}(T)$. It can be calculated analytically by a straightforward use of the corresponding equation of motion, obtained in section 2 . While the numerical method consists in the following centred second order scheme :

$U_{f}\left(T+\frac{\Delta T}{2}\right)=\frac{X_{w}(T+\Delta T)-X_{f}(T)}{\Delta T}+\mathrm{O}\left(\Delta T^{2}\right)$

where $U_{f}$ denotes the front velocity and subscript $w$ is used for $b, f s$ and $f l$ when referring to the front of back wave or positive wave in the short time regime and in the long time regime, respectively. The results obtained using both methods are concordant

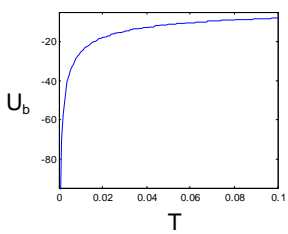

Figure 9: Time variation of the velocity of the back wave

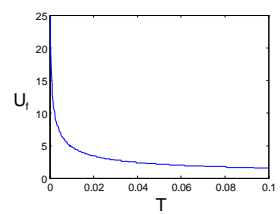

Figure 10: Time variation of the velocity of the positive wave in the short time regime

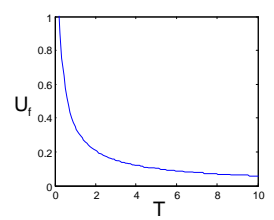

Figure 11: Time variation of the velocity of the positive wave in the long time regime

These graphs clearly show that for each wave, the velocity is time decreasing and tends to an asymptotic value which for the positive wave characterizes a 1D film lubrication.

\section{F. Comparison of the numerical results with the analytical ones}

Dam-break flow belongs to the general class of gravity currents; so the solution depends on the time scale [11]. First of all, the inertial regime, characterized by a fixed height at the dam-site holds immediately after the dam collapse [1]. Then, a solution dominated by viscous effects appears and tends to an asymptotic form. The solution sought here will give the analytical expression for a short time $(T<<1)$ and a long time $(T>>1)$ viscous solutions, as well as the different dynamic characteristics. Sedov [11] describes the method of investigating similar solutions of equ. (9) by means of a phase plane formalism. In fact, this equation of motion can be tackled by assuming a solution of the form

$$
H(X, T)=\Omega(T) \Psi(\lambda) \text { where } \lambda=\frac{X-\phi(T)}{P(T)} \quad(41)
$$

Let $X_{b}(T)$ denote the front of the back wave and $X_{f}(T)$ the front of the positive wave. If $T_{c}$ denotes the time where the back wave front reaches the rear wall, the short time regime corresponds to a viscous solution such that $T \leq T_{c}$. While, for larger time, $H$ is everywhere less than 1 . So, a solution should be sought such that

$$
H\left(X_{b}(T), T\right)=\left\{\begin{array}{ccc}
1 & \text { if } & T \leq T_{c} \\
H(-1, T) & \text { if } & T \geq T_{c}
\end{array}\right.
$$


$X_{b}(T)=\left\{\begin{array}{ccc}X_{b}(T) & \text { if } & T \leq T_{c} \\ -1 & \text { if } & T \geq T_{c}\end{array}\right.$

Two regimes can now be identified, which correspond to two different physical mechanisms of reservoir emptying. The short time solution is such that far downstream from the dam, the fluid seems to be at rest at a depth $h_{0}$, so that the reservoir's length $l_{0}$ has no effect on this flow regime, while for the long time solution, the flow only retains the initial (non dimensional) volume of the reservoir $V=1 L$ and not the details of its initial geometry.

To find the short time solution, $w$ estate that the information affects the fluid contained between $X_{f}(T)$ and $X_{b}(T)$, this suggests to take

$P(T)=X_{f}(T)-X_{b}(T), \phi(T)=X_{b}(T)$

Introducing equs. (13)-(15) in the equation of motion (9), we get

$-\frac{d}{d \lambda}\left[\frac{d \Psi^{4}}{d \lambda}\right]+P^{\prime} P \lambda \frac{d \Psi}{d \lambda}+X_{b}^{\prime} P \frac{d \Psi}{d \lambda}=0$

For the long time solution, we state that, at $T=T_{c}$, the front of the negative wave reaches the rear wall $\left(X_{b}=-1\right)$, so for the long time solution, we can take $\psi(T)=-1$ and $P(T)=X_{f}+1$

Then, introducing equ. (24) in the equation of motion (9), we get

$-\frac{d}{d \lambda}\left[\frac{d \Psi^{4}}{d \lambda}\right]+\frac{\Omega^{\prime} P^{2}}{\Omega^{4}} \Psi-\frac{P^{\prime} P}{\Omega^{3}} \lambda \frac{d \Psi}{d \lambda}=0$

When computing the time and abscissa variation of the fluid height in the short time viscous regime, we observed that the results from the numerical method (fig. 13) were generally greater than those obtained from the analytical method (fig. 12). Meanwhile, the results from the two methods were concordant with a relative difference less than $15 \%$. The same remark holds for the long time viscous regime but the relative difference being now less than $2 \%$.

To state on the accuracy of the methods used, we zoomed the different graphs obtained in the short time regime, on the dam site. We observed that the graphs observed from the numerical method (fig. 15) intersected at a point which is more close to the one obtained experimentally, than in the analytical case (fig. 14). So, the numerical method seems more accurate than the analytical one.

\section{Conclusion}

The flow regimes of the horizontal viscous dam-break flow are well known from experimental studies. At initial time (when the dam collapses), the fluid is released downstream (positive wave), while a negative wave propagates upstream. The flow is inertial (Ritter's solution) until the back wave reaches the fixed rear wall. Then, the viscous forces become higher than the inertial ones and a short time viscous regime takes place until. In this regime, the flow height at dam site has a (fix) characteristic value. As the reflected wave overtakes the positive wave, the long time or asymptotic regime takes place. The present study considered the modelling of these two viscous flow regimes.

Applying the conservation of mass and momentum with the shallow water approximation, an equation of motion was derived and made non dimensional, when the viscous forces were assumed to be the dominant ones. It was of porous medium type and similar solutions built analytically.

Then, the problem was considered numerically. The previous equation of motion was approximated using an explicit finite difference method. The stability and convergence of the computations were insured using a criteria based on heuristic approach. The very good agreement between the numerical and the analytical solutions showed the consistence of the numerical scheme for both short time and long time solutions. The time evolution of the abscissa and velocities of the different front waves were determined, as well as the different characteristic heights.

\section{References:}

[1] Ritter, A.: Die fortpflanzung der wesser wellen. Ver Deutsch Ingenieure Zeitschrift, Vol.36, N³3, pp. 947954, 1892 (in German)

[2] Gill, M.A.: "Dam-break problem." Encyclopedia of fluid mechanics, 6, N.P. Cheremisinoff, eds., Gulf, Houston (Texas), 1429-1473, 1987

[3] De Saint-Venant, B.: Théorie du mouvement non permanent des eaux. C. R. Acad. Sci., vol. 73(3), 147154, 1871 (in French)

[4] Faure, J. And Nahas, N.: Etude numérique et expérimentale d'intumescences à forte courbure du front. », La Houille Blanche, vol. 16(5), 576-587, 1961 (in French)

[5] Hunt, B.: Newtonian fluid mechanics treatment of debris flows and avalanches. J. Hydr. Div. (ASCE), vol. 120(12), 1350-1363, 1994

[6] Nsom, B., Debiane, K. and Piau, J.M.: Bed slope effect in the dam-break problem. J. Hydr. Res. (IAHR), vol. 38(6), 459-464, 2000

[7] Nsom, B.: Horizontal Viscous Dam-Break Flood: Experiments and Theory. J. Hydr. Eng. (ASCE), vol. 128(5), 543-546, 2002

[8] Schwarz, L.W.: Viscous flows down an inclined plane: Instability and finger formation. Phys. Fluids A, vol. 1(3), 443-445, 1989

[9] Zoppou, C. and Roberts, S.: Explicit Schemes for Dam-Break Simulations. J. Hydr. Eng.(ASCE), vol. 129(1), 11-34, 2003

[10] Shigematsu, T., Liu, P.L.F. and Oda, K.: Numerical modeling of the initial stages of dam-break waves. J. Hydr. Res. (IAHR), vol. 42(2), 183-195, 2004

[11] Simpson, J.E.: "Gravity Currents in the Environment and the Laboratory “, John Wiley \& Sons, New York, 1987 